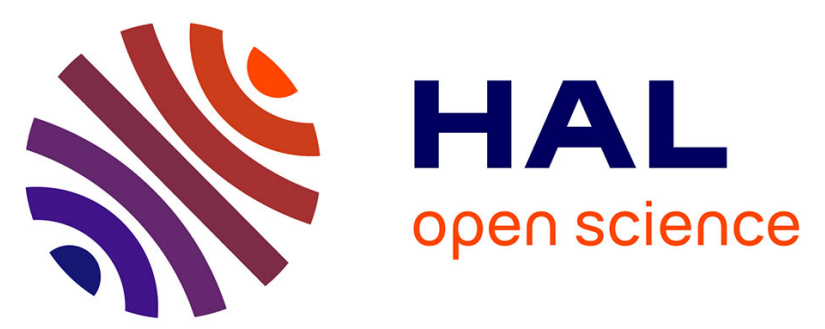

\title{
ANALYSE DYMIQUE ELASTO-VISCOPLASTIQUE DE L'HÉTÉROGENÉITE DE LA DÉFORMATION PLASTIQUE DE CISAILLEMENT
}

\author{
C. Fressengeas
}

\section{- To cite this version:}

C. Fressengeas. ANALYSE DYMIQUE ELASTO-VISCOPLASTIQUE DE L'HÉTÉROGENÉITE DE LA DÉFORMATION PLASTIQUE DE CISAILLEMENT. Journal de Physique Colloques, 1988, 49 (C3), pp.C3-277-C3-282. 10.1051/jphyscol:1988339 . jpa-00227762

HAL Id: jpa-00227762 https://hal.science/jpa-00227762

Submitted on 1 Jan 1988

HAL is a multi-disciplinary open access archive for the deposit and dissemination of scientific research documents, whether they are published or not. The documents may come from teaching and research institutions in France or abroad, or from public or private research centers.
L'archive ouverte pluridisciplinaire HAL, est destinée au dépôt et à la diffusion de documents scientifiques de niveau recherche, publiés ou non, émanant des établissements d'enseignement et de recherche français ou étrangers, des laboratoires publics ou privés. 


\title{
ANALYSE DYNAMIQUE ELASTO-VISCOPLASTIQUE DE L'HETEROGENEITE DE IA DEFORMATION PLASTIQUE DE CISAILLEMENT
}

\section{FRESSENGEAS}

\author{
Laboratolre de Physique et Mécanfque des Matériaux, CNRS UA-1215, \\ Université de Metz, F-57045 Metz Cedex, France
}

\begin{abstract}
Résumé: La morphologie des bandes de cisaillement obtenues à très grande vitesse est étudiée au moyen d'un modèle élasto-viscoplastique tenant compte du couplage thermique, de la conduction thermique et des effets d'inertie. Les calculs fournissent la largeur et l'espacement des bandes, ainsi que leur temps critique de formation. L'interaction des bandes de cisailiement est etudiée.

Abstract: The morphology of adiabatic shear bands at very high strain rates is investigated through an elastic uiscoplastic model with thermal coupling, accounting for heat conduction and inertia effects. The calculations provide the bands width, spacing and critical growth time. Shear bands interactions are explored.
\end{abstract}

\section{1- 1 introduction.}

fux trés grandes vitesses de déformation, la croissance rapide de la déformation plastique engendre un grand nombre d'amorces de bandes de cisaillement; la morphologie de la déformation plastique dépend alors à la fois de la vitesse de formation des bandes et de la célérité des ondes de détente qui trouvent leur origine dans ces bandes, et qui se propagent dans le matériau adjacent. On peut dire de manière assez imprécise, que dans la région atteinte par ces ondes, aucune nouvelle bande ne peut se développer jusqu'à son terme; c'est seulement dans une zone non encore atteinte par les ondes de détente, et se déformant encare plastiquement que de nouvelles bandes peuvent se former et croitre sans interaction avec la bande primaire (1).

De nombreux etudes ont fourni des informations sur les conditions de formation des bandes, ainsi que sur leur vitesse de développement $(2,3,4,5,6)$. Cependant ces travaux $n$ 'ont pas abordé le probleme de la propagation de l'onde plastique, principalement en raison du fait qu'ils se situent dans un domaine de vitesses où le caractere dynamique n'est pas suffisamment prononcé.

Parallelement, un certain nombre d'auteurs ont entrepris l'étude du phénomene de propagation de l'onde de détente(1,7,8), au prix toutefois de simplifications considérables des mécanismes de l'instabilité à l'oeuvre dans la bande elle-meme; ceux-ci sont inclus seulement a titre de condition à la limite du problème de propagation.

Le travail presenté $\mathrm{ici}$ est une tentative pour éviter ces deux types de simplifications; il a pour but de fournir simultanément et de maniere consistente les caractéristiques de la bande (largeur de bande, temps critique...) et de ses interactions dynamiques aves les bandes voisines (celérité de l'onde plastique, espacement des bandes..) dans le contexte des tres grandes vitesses obtenues par exemple sous choc, et de l'ordre de $10^{7}-10^{8} s^{-1}$

2- Formulation du modele

On considere une tranche de matériau d'épaisseur constante $2 h$ dans la direction $\bar{y}$, s'étendant à l'infini dans les directions $\bar{x}$ et $\bar{z}$. On suppose que tous les deplacements dans les directions $\bar{y}$ et $\bar{z}$, et toutes les dérivées par rapport à $\bar{x}$ et $\bar{z}$ sont nuls. La vitesse $\bar{u}$ des particules 
dans la direction $\bar{x}$ est ainsi une fonction de $\bar{y}$ et du temps $E$, et l'on obtient une formulation unidimerisionnelle. En raison de quelque pluctuatiuon initiale du champ de contraintes et de températures locales, on suppose qu'une bande tende à se former au centre de la zone etudiée; pour des raisons de symétrie, l'origine du repere inertiel de réference est fixée en ce point, et seule une demi- tranche est considérée. Le champ de vitesses est soumis aux conaitions à la limite: $\bar{u}(a, E)=0, \bar{v}(h, E)=V, t 20$
$(2-1)$

où $V$ est la vitesse supposée constante du bord supérieur. Les conditions à la limite thermiques sont supposees adiabatiques, de sorte que:
$\frac{\partial \bar{E}}{\partial t}(\theta, E)=\frac{\partial \bar{\theta}}{\partial t}(h, E)=0, E \geqslant 0$
$(2-2)$

où $\overline{\bar{\theta}}$ désigne la température. On suppose que la witesse de déformation totale $\bar{\gamma}$ est la somme de la vitesse de déformation plastique $\bar{\gamma}_{p}$ et de la vitesse de déformation élastique $\bar{\gamma}_{e}$ : $\bar{\gamma}=\bar{y}_{p}+\bar{Y}_{e}$

Le comportement du matériau est spécifié par ıne loi élastique linéaire et par la loi empirique thermo-viscoplastique suivantes:

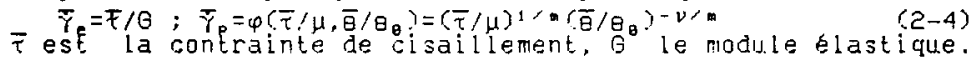

des des constantes qui caractérisent respectivement la uiscosité la sensibilité à la température et à la vitesse; $\theta_{0}$ désigne la valeur initiale uniforme de la température. La masse volumique du matériau est notée $\rho$; dans le but d'obtenir des équations non dimensionnelles, on introduit les variables sans dimension:

$$
\begin{aligned}
& \mathrm{t}=\dot{\gamma}_{\theta} E, \quad \dot{\gamma}=\bar{\gamma} / \dot{\gamma}_{\theta}, \dot{\gamma}_{e}=\bar{\gamma}_{e} / \dot{\gamma}_{\theta}, \dot{\gamma}_{p}=\bar{\gamma}_{p} / \dot{\gamma}_{\theta}
\end{aligned}
$$

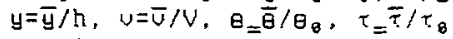

$\dot{\gamma}_{0}$ est la vitesse de déformation homogene initiale, liée à $\tau_{0}$, $V$ et $h$ par :

$$
\tau_{\theta}=\mu \dot{\gamma}_{\theta}, \dot{\gamma}_{\theta}=V / h \quad(2-6)
$$

Les équations áfinissant le modèle incluent outre les lois de comportement déjà spécifiées les équations dynamique et thermique; elles s'ecrivent alors:

$$
\begin{aligned}
\rho_{0} \frac{\partial y}{\partial t} & =\frac{\partial \tau}{\partial y} \\
\frac{\partial \gamma_{p}}{\partial t} & =\varphi(\tau, \theta) \\
\frac{\partial \tau}{\partial t} & =G_{\theta}\left(\frac{\partial u}{\partial y}-\varphi\right) \\
\frac{\partial \theta}{\partial t} & =k \partial^{2} \theta+\alpha \tau \varphi
\end{aligned}
$$

Les quatre constantes. $\beta_{\theta}, \hat{F}_{0} k$ et $\alpha$ sont des paramères de similitude; $\theta_{\theta}=8 / \tau_{B}$ caractérise l'élasticité du matériau, et $\rho_{\theta}=\rho v^{2} / \tau_{0}$ les effets d'inertie en les comparant à la contrainte d'écoulement. Les effets thermiques sont caracterisés par $k=K / \mathrm{Ch}^{2} \dot{\gamma}_{\theta}$ et $\alpha_{B} B \tau_{\theta} / \mathrm{C \theta}_{0}$ où $K, C$ et $\beta$ sont respectivement le coefficient de conduction themique, la capacite thermique volumique et le coefficient de couplage thermo-mécanique.

On suppose l'existence d'un écoulement plastique homogene initial: $u(y, 0)=y, \gamma,(y, 0)=0$ $(2-11)$

Deux problemes différents sont traités dans la suite; ils sont specifiés par les deux conditions initiales qu'il reste a formuler; dans le premier cas (Paragraphe 3 ), on suppose 1 'existence d'une fluctuation de contrainte et de temperature $d^{\prime}$ amplitude $\varepsilon$ et d'extension $d$ : $\theta(y, 0)=1+\varepsilon(1+\cos \pi y / d) / 2, \quad \theta \leqslant y \leqslant d$
$\theta(y, 0)=1, d \leqslant y \leqslant 1 ; \tau(y, \theta)=a^{\nu}(y, 0)$

Dans le contexte des tres grandes uitesses, de telles fluctuations sont des variations intragranulaires. Au paragraphe 4 , on étudie les interactions de bandes initiées par deux défauts de même amplitude, de même extension et espacés d'une distance 1: 
$\theta(y, \theta)=1+\varepsilon_{1}(1+\cos \pi y / d) / 2+\varepsilon_{2}(1+\cos (1-y) / d) / 2 ; \tau(y, \theta)=\theta^{n}(y, \theta)$ $\varepsilon_{1}=0, y 2 d ; \varepsilon_{1}=\varepsilon, y \leqslant d: \varepsilon_{2}=0,|1-y| \lambda d ; \varepsilon_{2}=\varepsilon,|1-y| \leqslant d \quad(2-13)$

La solution du systéme d'équations aux dérivées partielles (2-7,2-10) soumis aux conditions initiales (2-12) ou (2-13) et aux conditions aux limites $(2-1,2-2)$ est de razure numérique; elle fait appel a la méthode de Galerkin mise en oeuure a l'aide d'élements finis.

3- Morphologie des bandes de cisaillement.

Nous utilisons les paramétres mécanlques fournis par ASAY-CHHABILDAS (10) et GRADY - KIPP (1), de manière a comparer nos prévisions aux résutats expérimentaux obtenus par ces auteurs avec un alliage d'aluminium 6061-T6 soumis à une déformation dưe a un impact de plaques $\rho=2700 \mathrm{~kg} / \mathrm{m}^{3}, G=2,7510^{4} \mathrm{Mpa}, \tau_{B}=500 \mathrm{Mpa}, \nu=-4,(3-1)$ $C=2,4310^{6} \mathrm{~J} / \mathrm{m}^{3} \mathrm{~K}, \quad K=235 \mathrm{~W} / \mathrm{mK}, \beta=1$

La température, la vitesse de deformation homogenes de réference et l'épaisseur de la tranche étudiée sont respectluement $B_{8}=300 \mathrm{~K}, \dot{y}_{8}=5,10^{7} \mathrm{~s}^{-1}, \mathrm{n}=5,10^{-6} \mathrm{~m}$

Bien que le parametre de sensibilité à la vitesse m ne soit pas spécifié par ces auteurs, nous supposons un régime visqueux à ces vitesses; la valeur $\mathrm{m}=0.01$ parâ̂t raisonnable pour cet alliage c voir KLOPP et al. (9)) et est adoptée dans la sulte. Les paramètres de similitude sont: $\rho_{0}=0.338, \quad \theta_{0}=55 ., \quad k=0.074, \alpha=0.686 ;$ ils sugserent que les effets dynamiques et de couplage sont importants car $p_{0}$ et a sont $O(1)$; le processus est quasi- adiabatique à l'échelle de la tranche car $k$ est voisin de $\theta$. La distribution de vitesses de déformation calculée pour $\varepsilon=10^{-2}$ et $d=250.10^{-9} \mathrm{~m}$ est représéntése sur la figure 1 .

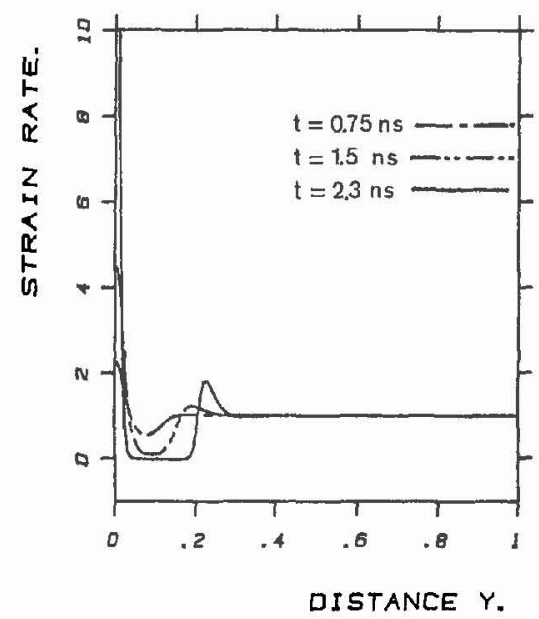

Figure 1: Distribution de la vitesse de déformation

On peut distinguer trois zones: la bande de cisaillement, ou la vitesse de deformation se localise de plus en plus pres du centre, puis une zone quasi rigide qui se développe à partir de la bande dans le matériau adjacent, et enfin une troisième région se déformant encore plastiquement de mani ère homogène. Cette dernière est séparke de la zone quasi rigide par le front d'onde plastique marqué par un accroissement secondaire de la vitesse de déformation. La déformation plastique 
homogene atteinte à l'extérieur de la bande est de l'ordre de $10 \%$. La célérité de l'onde est gouvernée par le paramètre d'inertie $\rho_{0}$; en effet deux calculs differents menés pour les valeurs $p_{0}=0.338$ et 0.216 ( les autres parametres etant maintenus constants) montrent que I'onde est plus rapide dans le second cas, et que la zone dechargée est plus étendue ( voir aussi $1,7,8$ ). Une seconde consequence de cette diminution

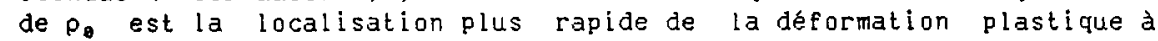
l'interieur de la bande ( voir aussi 5,6); l'interprétation est double: pour un parametre d'inertie fort, l'apport énergétique extécieur est dauantage affecté à l'entretien de la déformation plastique homogene, car la zone déchargée est moins étendue. Il en résulte que l'énergie cinétique et la uitesse de la zone rigide d'une part, le gradient de vitesse dans la bande d'autre part sont moindres. En second lieu, plus d'inertie entraine, à apport d'énergie cinétique donné, une augmentation de vitesse moindre pour la zone rigide, et par suite un accroissement plus faible de la uitesse de déformation dans la bande. Ainsi le délai apporté a la lacalisation ya de pair avec le rétrécissement de la zone de matériau déchargé. L'espacement des bandes est lié étroitement a l'extension de la zone déchargée; en effet, lorsque les ondes plastiques de détente émanant de deux bandes se rencontrent, elles définissent une région dans laquelle aucune nouvelle bande ne peut se développer indépendamment. Ainsi, le double de la zone dechargée a la localisatian fournit une estimation $D_{y}$ de l'espacement entre bandes(1). Ce point sera discuté plus avant au paragraphe 4. Nous définissons numériquement le temps de localisation $t_{e}$ par: $\dot{\gamma}\left(\theta, t_{e}\right) / \dot{\gamma}\left(1, t_{e}\right)=10$., et la largeur de bande a par: $\forall y$ $a \leq y \leq a / 2, \quad \dot{\gamma}\left(y, t_{e}\right) \geqslant \dot{\gamma}\left(1, t_{p}\right)$. Les valeurs numeriques obtenues a partir de ces critères sont:

$$
E_{e}=2.3 \mathrm{~ns}, \bar{a}=255 \mathrm{~nm}, \bar{D}_{4}=4.3 \mu \mathrm{m}
$$

Les études au microscope electronique a transmission des fragments récupérés $(1,10)$ indiquent que la déformation plastique est localisée dans un réseau. de bandes de cisaillement de largeur: 100-300 nm, espacées de 2 a $10 \mu \mathrm{m}$ : le temps total de deformation, qui est aussi le temps de montée de l'onde de choc, est de l'ordre de 1 a 2 ns. Nos préuisions (3-3) sont compatibles avec ces valeurs experimentales, ce qui conforte l'interprétation thermo- dynamique proposée par (1) pour ce réseau de bandes.

Nous avons fait varier les fluctuatinns initiales, a energie ou a amplitude $\varepsilon$ constante: dans chaque cas, un minimum apparât pour le temps de localisation: une bande tropétroite est stabilisée par la diffusiuité thermique, une bande trop large l'est par les effets d'inertie. De cet équilibre entre effets de conduction et effets d'inertie, émerge une fluctuation optimum: les bandes qui en résultent dominent la morphologie de la déformation plastique, car les ondes de détente qu'́lles génèrent arrêtent le développement de celles qui se developpent plus lentement. Les morphologies optimum calculeses sont. caractérisées par: $E_{2}=2.2 \mathrm{~ns}, \overline{3}=255 \mathrm{~nm} . \bar{\pi}_{10}=4.15 \mu \mathrm{m}$ à énergie constante, et: $E_{p}=2.1 \mathrm{~ns}, \overline{\mathrm{a}}=260 \mathrm{~nm}, \bar{D}_{u}=4.30$ um à amplitude coristante. Elles sont très proches des résultats indicatifs (3-3). et sont compatibles avec les observations experimentales.

La largeur de bande est remarquablement insensible a la taille des différentes fluctuations initiales imposés; lorsque ces dernieres uarient de $150 \mathrm{~nm}$ a $1 \mu \mathrm{m}$. la largeur de bande est trouvée entre 250 et. $275 \mathrm{~nm}$. Par suite elle est davantage gouvernée par un equilibre entre les phenomènes physiques en présence que par la taille des défauts initiaux; les calculs ci-dessus suggerent que la diffusion thermique blargit les bandes issues de fluctuations étroites, et que les effets 
dynamiques rétrécissent les bandes découlant de fluctuations trop larges. La largeur de bande optimum, liee au temps de localisation minimum se situe vers $255-260 \mathrm{~nm}$. Remarquons cependant ici que d'autres facteurs (transformations de phase..) peuvent avoir une influence considerable sur la largeur de bande.

4- Interactions des bandes de cisaillement.

Mous utilisons maintenant les conditions initiales (2-13); il résulte de nos calculs que la tranche de matériau considérée peut être divisée en trois régions distinctes. Une bande secondaire, générée a la distance 1 de la bande primaire placée à l'origine, se developpe jusqu'a son terme sans interaction avec la bande primaire si $1 \geqslant D_{u}$ : ceci définit la zone de "non interaction". Dans une seconde région: $D_{e} \leqslant 1<D_{u}$, une bande secondaire crôt également jusqu'à localisation complete, mais non sans interaction avec la bande primaire; lorsque le point de nucleation de la bande secondaire est déplacé vers $D_{R}$, son temps de localisation $t_{R}$ augmente, jusqu'à devenir infini pour $D_{2}$. Pour les valeurs $(3-1,3-2)$, nous auons obtenu $\tilde{D}_{e} \cong 1.25 \mu \mathrm{m}$ en faisant décroftre 1 . La Pigure 2 fournit un exemple relatif à cette zone d'interaction pour $I=1.75 \mu m$.

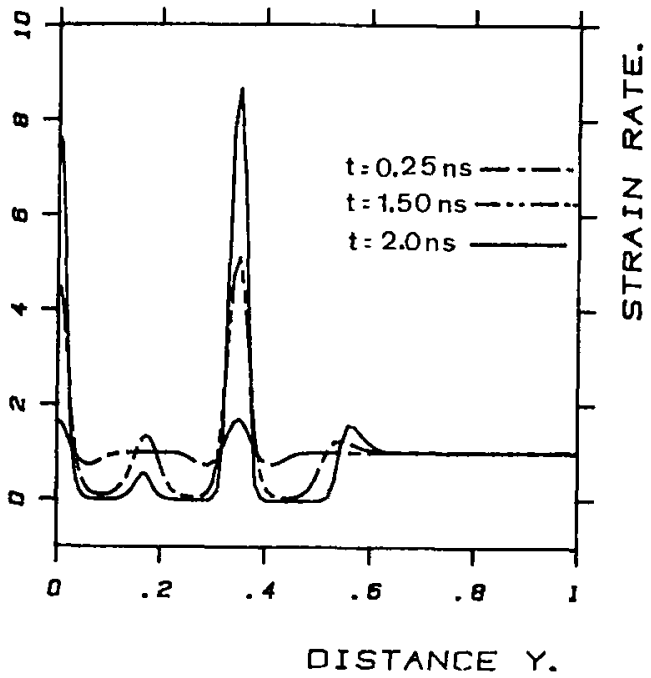

Figure ?: Bande primaire freiné:

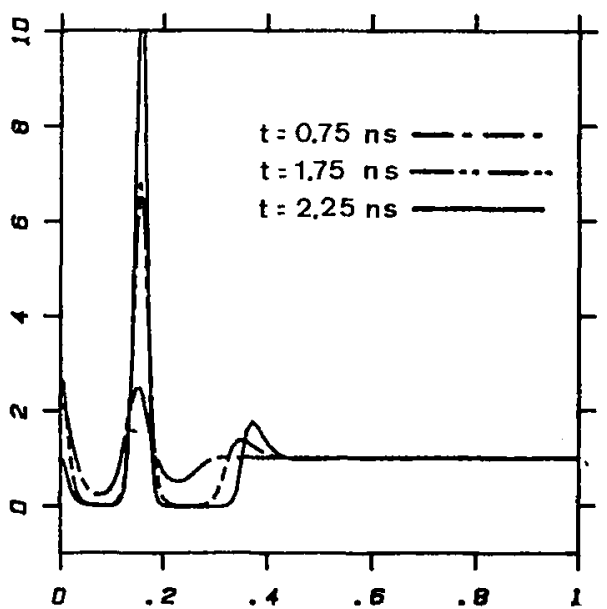

DISTANCE Y.

Figure 3: Bande primaire arreté.

La troisième région dans laquelle l'interaction provoque l'arret de l'une des bandes est définie par $a \leqslant 1<D_{e}$; on peut en voir un exemple sur la figure 3 , pour $T=0.75 \mu \mathrm{m}$. Après une période initiale de croissance, la bande primaire est "aualée" par l'onde de détente secondaire. Ces résultats suggerent que l'espacement entre bandes peut devenir aussi petit que $1.25 \mu \mathrm{m}$, et cependant conduire a la localisation complète, si l'apport extérieur d'energie est suffisant pour entretenir des bandes aussi nombrelses. Si l'on suppose que des fluctuations sont disponibles en tout point pour $y$ initier une bande de cisaillement, alors la distance $D_{v}$ est la distance maximum entre bandes; par suite l'espacement préuu entre bandes se situe entre 1.25 et $4.3 \mu \mathrm{m}$, ce qui est largement compatible avec la tendance observée: $2-10 \mu \mathrm{m}$. Une vitesse nominale $\dot{\gamma}_{\theta}$ plus faible entrainerait un ecart $D_{e}-D_{u}$ plus large. 
$5=$ Résumé; Conclusions.

Cette étude est consacrée à la déformation à très grande uitesse des matériaux, avec application à un alliage d'aluminium 6061-T6; un modele dynamique elasto-viscoplastique avec couplage et conduction thermique est proposé pour décrire la formation $d$ un réseal de bandes de cisaillement intragranulaires. Les principaux résultats obtenus sont les suivants:

- Les effets d'inertie gouvernent la morphologie de la déformation plastique hétérogène; ils stabilisent la déformation homogène en repoussant la localisation dans les bandes de cisalllement à de plus grandes déformations plastiques. Cependant lorsque de telles deformations sont atteintes, la localisation a Lieu dans des bandes plus rapprochees et plus nombreuses que dans une sollicitation moins dynami que.

- Le temps critique de localisation et la largeur de bande résultent d'un équilibre entre les effets de conduction thermique et les effets d'inertie: la diffusion de la chaleur tend a augmenter la largeur des bandes formées à partir de fluctuations trop étroites; la détente due aux effets d'inertie tend au contraire a rétrécir les bandes issues de fluctuations trop larges.

- Le temps de formation. la largeur et l'espacement des bandes calcules sont en bon accord auec les ualeirs observées.

Remerciements: Ce travail a été accompli pour partie au Center for Materials Science. Los Alamos National Laboratory, U.S.A. lors d’un séjour sabbatique de l'auteur, et financé par le Department of Energy. Le support financier de la Direction des Recherches Etudes et Techniques (Contrat 86/1388) a également été apprécié. L'auteur remercie J.R. KLEPACZKO de son aide et. de ses nombreuses suggestions.

Réferences:

1)GRADY,D.E., K.PP, M.E., J.Mech.Phys.SOL .35,95, 1987.

2)CULVER.R.S. Metallurgical Effects of High Strain Rates (ed.R.W.RHODE, B.M.BUTCHER, J.R.HOLLAND, C.H.KARNIES), p519, Plenum N.Y., 1973.

3)CLIFTON,R.J.. Adiabatis Shear; Report. NMAB-356 NRC Committee on Material Response to Ultrasonic Loading Rates, 1978.

4)BAI ,Y.L. . J.Mech.Phys.SOL .30,195,1982.

5) MOLINARI, A. , J.Mer. Th. Appl , 4, 659, 1985.

6)FRESSENGEAS,C., MOLINARI , A. J.Mech.Phys.Sol .35,185, 1.987.

7)MOTT,N.F., ProC.ROY.SOC., A189,300,1947.

8)LEE,E.H., Energetics 111 (Ed. W.MUELLER, M.SHAW), p85, Gordon and Breach.N.Y., 1967.

9)KLOPP,R.W., CLIFTON,R. I., SHAWKI, T. . ., Mech.Mat, ,4,375, 1985.

10)ASAY.J.R., CHHABILDAS.L.C.. Shock waves and High Strain Rate Phenomena in Metals (Ed M.A.MEYERS, L.E.MURR),P417,Plernum N.Y.,1981. 